\title{
Different Forms Effect of Single Complementary Metamaterial Structure on Square Patch Antenna
}

\author{
A. Bendaoudi and R. Naoum \\ Laboratory of Telecommunication and Digital Signal Processing. University of Sidi Bel Abbès, 22000, Algeria
}

\begin{abstract}
In this paper, we propose four different shapes - square, circular, triangular, and rhombic- of single complementary double negative metamaterial (C-DNM) structure incorporated into the square patch antenna. We compare the performance of the proposed structure with a conventional patch antenna, in a same parametric analysis with HFSS simulator. The operating frequency of this antenna is around $12 \mathrm{GHz}$ for Xband applications. The parameters that considered in these works are resonant frequency, reflection coefficient, directivity, bandwidth and radiation pattern. The focusing parameter is to achieve the better directivity gain and directional radiation pattern that obtained from the single double negative C-DNM patch antenna. The simulations show that the addition of C-DNM to square patch antenna provides better performance in terms of directivity $7.78 d B$ to $8.02 d B$ for square metamaterial.
\end{abstract}

Keywords: microstrips patch antenna, double negative metamaterial, directivity, radiation pattern, reflection coefficient.

\section{INTRODUCTION}

In 1967, Victor Veselago [1] showed theoretically that a medium whose permittivity and permeability are negative simultaneously had a negative refractive index. This dual requirement has long been difficult to achieve experimentally. In 2000, John Pendry [2] proposed an implementation using periodic metallic structures formed of concentric rings cut, called split-ring resonators (SRR) with a negative permeability, and a wire with continuous metal in low frequency negative permittivity. By bringing the two networks in a periodic composite structure, Pendry has realized medium proposed by Veselago. This medium then has a negative index around the resonant frequency of the resonators SRR.

One of the promising applications in this structure is the field of antennas based on metamaterials. The double negative metamaterial structure proposed will achieve a better reflection coefficient in the normal antenna. The structure can also improve the directivity gain of the antenna. In this context, the prospective study is to explore the possibility simulated of a square patch antenna based on the double negative matamatériaux. We propose two different double negative metamaterial, the first one is normal and the second one is a complementary cell. This cell is achieved by replacing the substrate material with the copper parts and parts of copper with a substrate material as shown in the figure below:

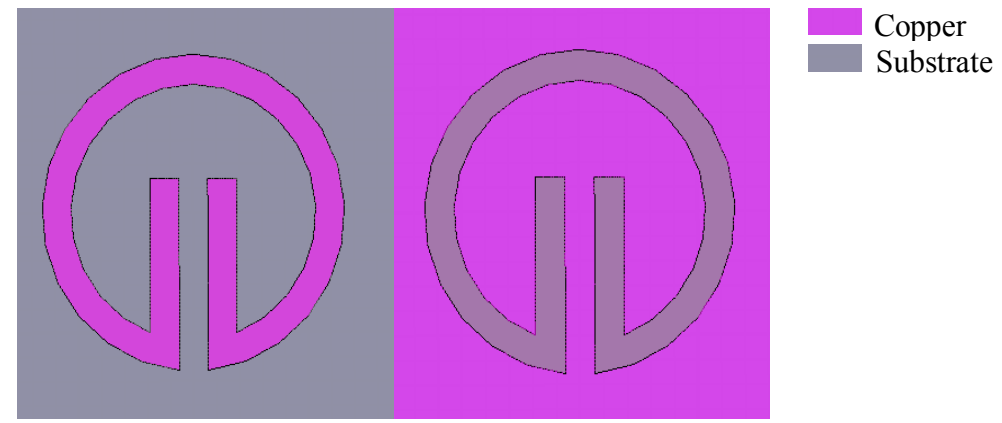

Fig 1. (a) Normal double negative metamaterial, (b) Complementary double negative metamaterial

The paper is organized as follows. In section II, the model of square patch antenna is given. Section III describes the proposed configuration of double negative metamaterial cell. Section IV shows single complementary double negative metamaterial had been incorporated into the square patch antenna. Section V presents the simulation results and the section VI concludes the paper.

\section{THE DESIGN OF SQUARE PATCH ANTENNA}

This design was proposed by reference [3] for military applications. The square patch antenna is constructed on dielectric substrate made of Taconic TLY-3 and fed by a coaxial cable having a characteristic 
impedance of $50 \mathrm{ohm}$ through the ground plane. All dimensions are shown in figure 2 with dimensions corresponding to resonance frequency around $12 \mathrm{GHz}$ and can be calculated using the following formula [4]:

$$
f_{1,0}=\frac{c}{2 L \sqrt{\varepsilon_{r}}}
$$

where $c$ is the speed of light in free space, $L$ is length and $\varepsilon_{\mathrm{r}}$ is the dielectric constant of the substrate.

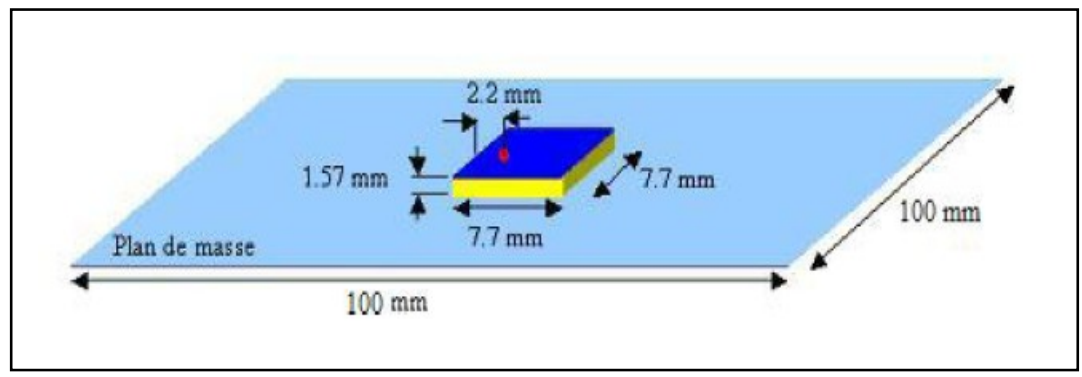

Fig 2. Dimensions of the square patch antenna [3]

\section{PROPOSED DOUBLE NEGATIVE METAMATERIAL CELL}

The application of double negative metamaterial (DNM) for improving the performance of the patch antenna is based on several parameters such as: the shape, the gap, the size and number of metamaterial. This paper focuses on the effect of different forms of DNM structure. The various shapes of complimentary DNM used in the simulation are: square, circular, triangular and rhombic and they have the same dimensions for the gap $\mathrm{G}=25 \mathrm{~mm}$, the width of the copper track $\mathrm{L}=2.63 \mathrm{~mm}$ and for the circular diameter is $2.63 \mathrm{~mm}$. The figure below shows the four forms of the DNM.

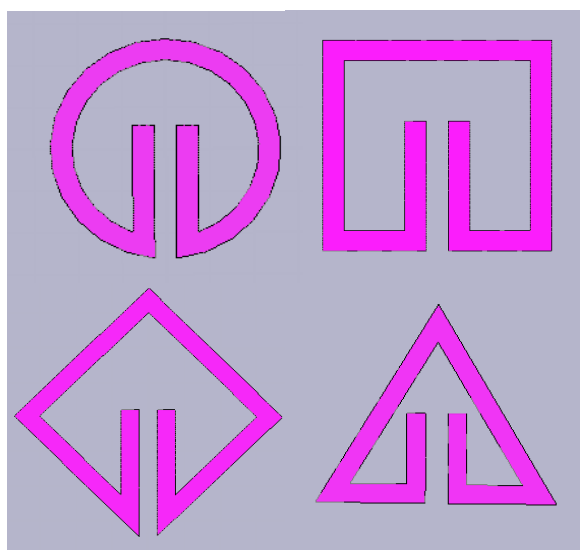

Fig 3. Single complementary double negative metamaterial structure with 4 different shapes - square, circular, triangular and rhombic

\section{ANTENNA INCORPORATED WITH SINGLE C-DNM}

Four different types of single complementary double negative metamaterial had been incorporated into the square patch antenna as is clear from Figure 4. The C-DNM is placed in the center of the square patch antenna and has resonance frequency is around $12 \mathrm{GHz}$. The figure shows one of the forms of C-DNM (circular) which is placed at the center of the square patch antenna. 

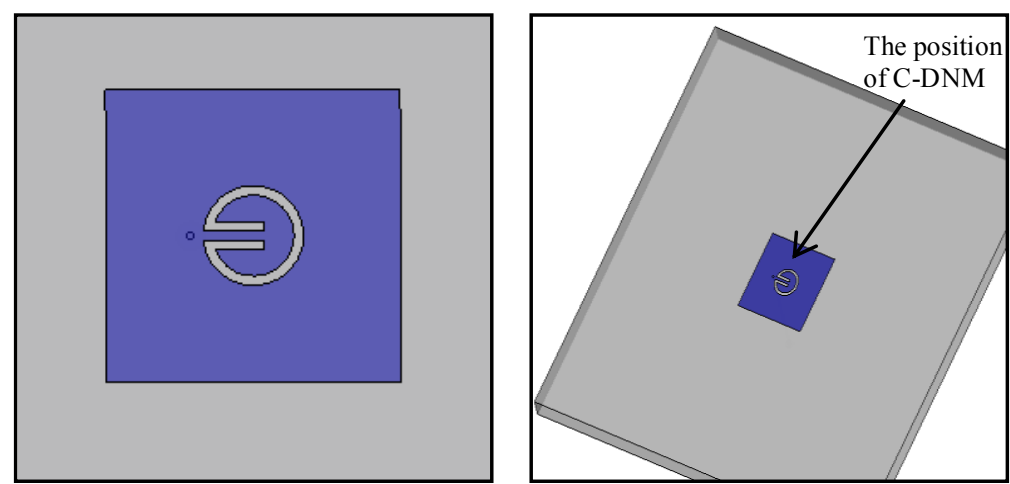

Fig 4. The incorporated C-DNM onto the center of the square patch antenna.

\section{Basic patch antenna design}

\section{RESULTS AND DISCUSSION}

\subsection{Reflection coefficient}

For the simulations we used HFSS. The coaxial feed source is powered by a waveport. The model area is surrounded by an absorbent box (radiation). In the following figure 5, we see the result of the reflection on the source (waveport) and represent the reflection coefficient curve. At $12.06 \mathrm{GHz}$ reflections is $-36.96 \mathrm{~dB}$ minimum, so $12.06 \mathrm{GHz}$ is the optimum operating frequency.

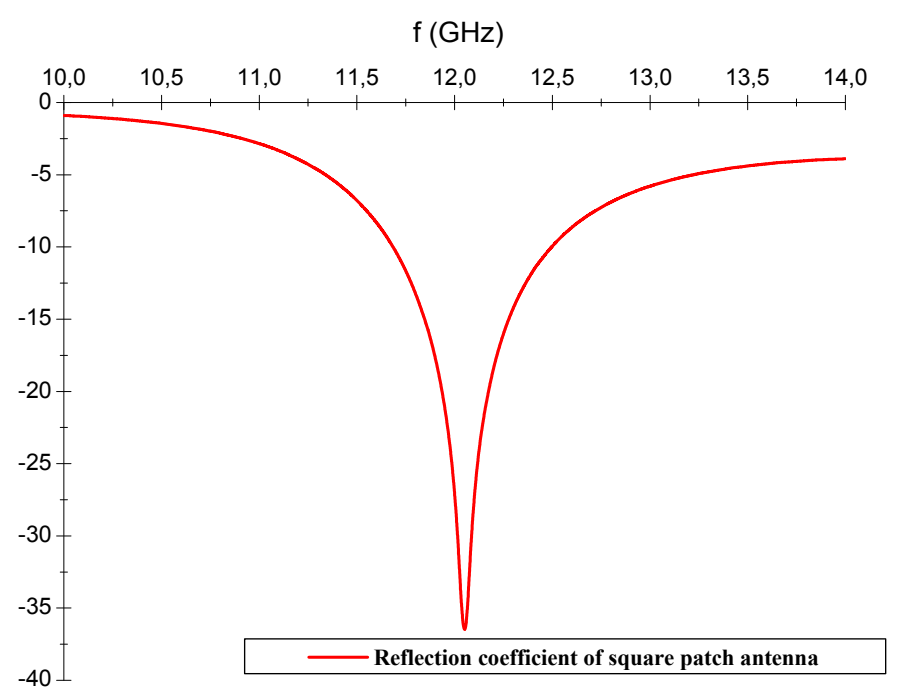

Fig 5. Simulated reflection coefficient (in dB)

\subsection{D-Directivity}

The figure 6 shows the directivity gain of the antenna in two different planes. We will use the following angles $\varphi$ and $\theta$ known spherical coordinates ( $\varphi$ represents the angle about the $\mathrm{X}$-axis in the XY plane; $\theta$ represents the angle to the $\mathrm{Z}$ axis). Then we look at the directivity in the $\mathrm{XZ}$ plane $\left(\varphi=0^{\circ}\right)$ and the $\mathrm{YZ}$ plane $(\theta$ $=90^{\circ}$ ), so the maximum directivity gain of antenna obtained from the graph is $7.78 \mathrm{~dB}$ in $0^{\circ}$ direction. 


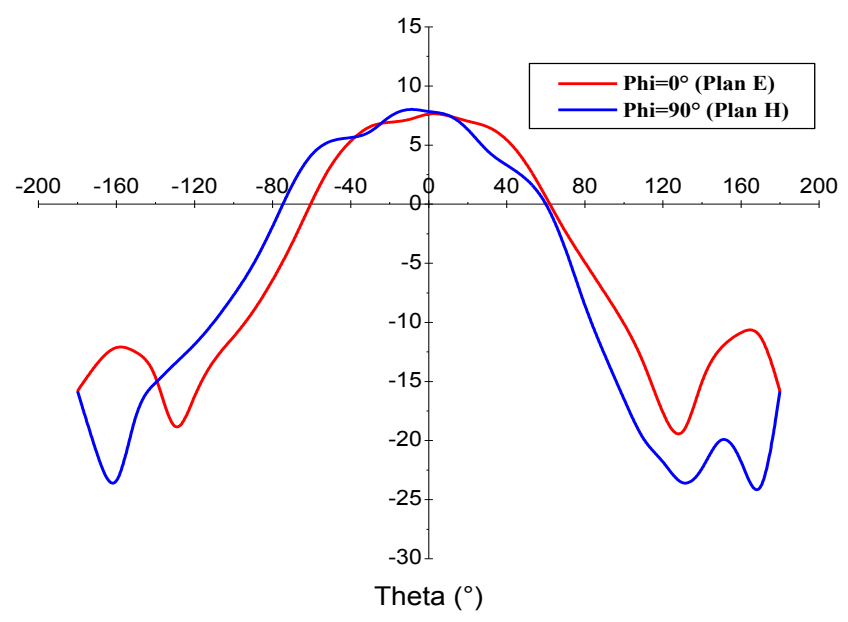

Fig 6. Simulated directivity (in $\mathbf{d B}$ )

\subsection{Radiation pattern}

The figure 7 shows the radiation pattern of square antenna. By observing the radiation pattern it seems that the pattern of $\left(\theta=0^{\circ}\right)$ is better than $\left(\theta=90^{\circ}\right)$. The Half-Power BeamWidth (HPBW) in the E-plane is $89 \mathrm{deg}$ and 69deg in the H-plane.

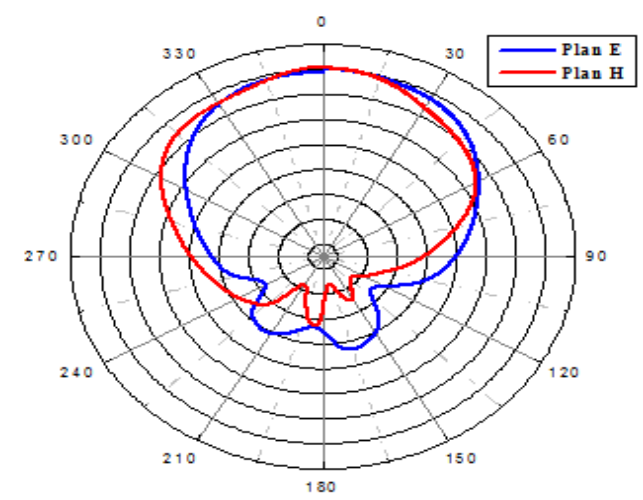

Fig 7. The radiation pattern around $12 \mathrm{GHz}$

\section{Square patch antenna design with C-DNM structure}

\subsection{Reflection coefficient}

Figure 8 shows the reflection coefficient performance of the square patch antenna with different shapes of single complimentary double negative metamaterial structure. From the observation, it shows that the addition of C-DNM had shifted the resonance frequency.

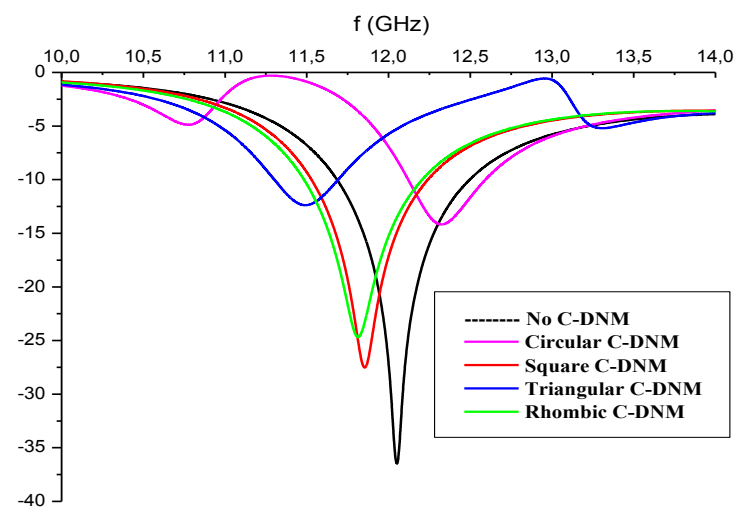

Fig 8. Simulated reflection coefficient (in dB) 
Figure 8 shows the results of reflection coefficient of antenna with and without complementary metamaterial load. In this figure, the antenna has a new resonance frequency.

\subsection{D-Directivity}

To see the effect of C-DNM in terms of directivity and compared with square patch antenna, we carried out the simulations shown in Figure 9.

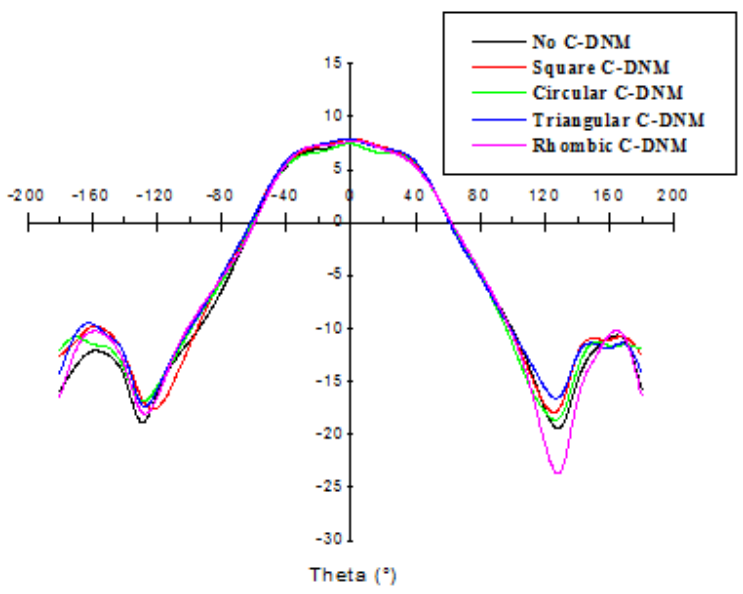

$\mathrm{Phi}=\mathbf{0}^{\circ}$

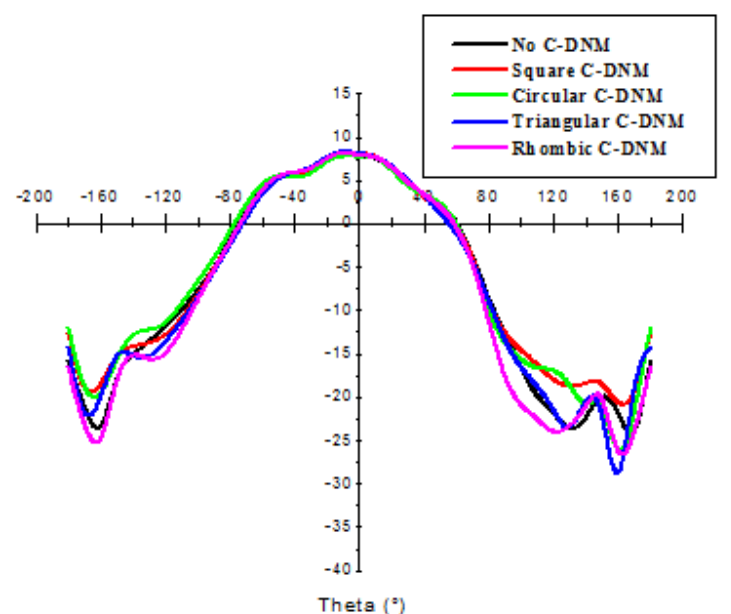

$\mathrm{Phi}=90^{\circ}$

Figure 9. Simulated directivity (in $\mathrm{dB}$ )

\subsection{Radiation pattern}

Simulation results of the radiation patterns of the antenna with and without comlementary metamaterial load are presented in figure 10 and (a) and (b) show both the E-plane and H-plane radiation patterns.

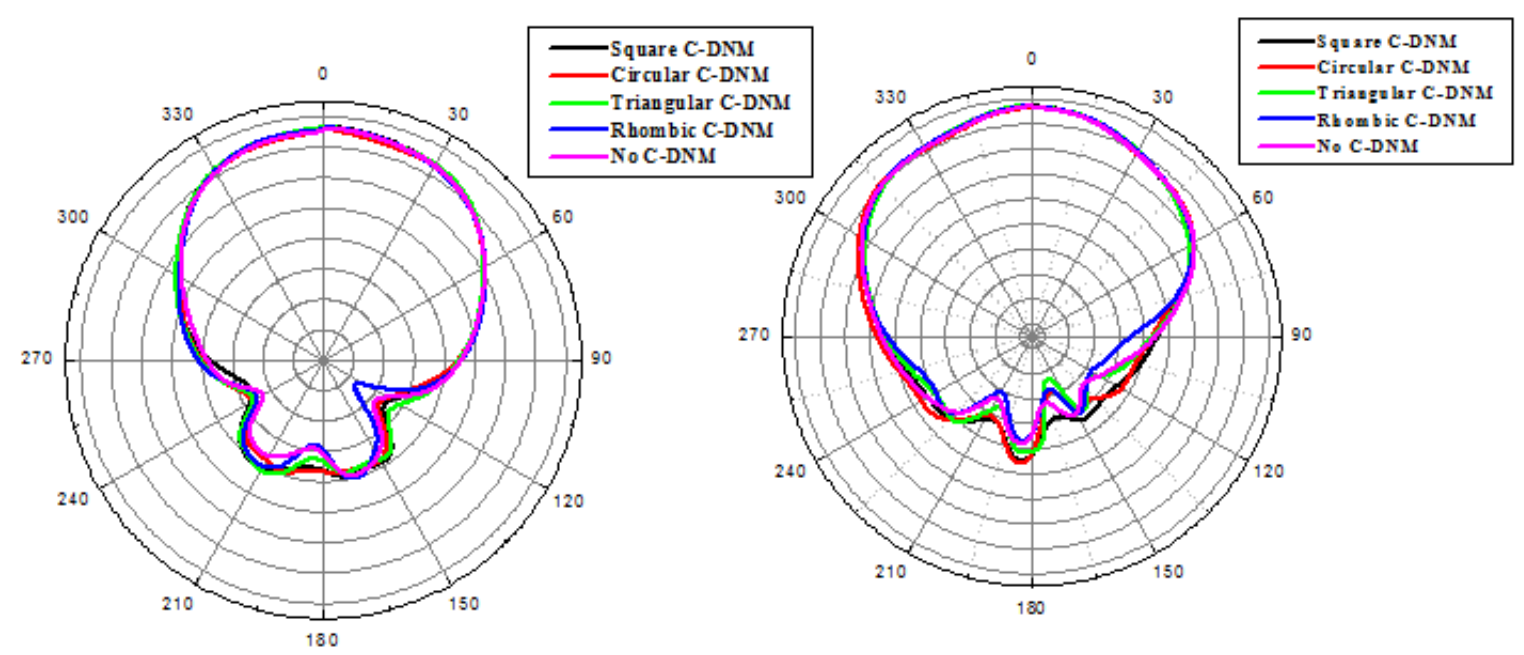

(a)

(b)

Fig 10. The radiation pattern around $12 \mathrm{GHz}$

Table 1: Comparison of parameter performance result between the normal patch antenna and with single C-DNM structure.

\begin{tabular}{|l|c|c|c|c|c|}
\hline 0. & $\begin{array}{c}\text { No C-DNM } \\
\text { structure }\end{array}$ & $\begin{array}{c}\text { Circular } \\
\text { C-DNM }\end{array}$ & $\begin{array}{c}\text { Square } \\
\text { C-DNM }\end{array}$ & $\begin{array}{c}\text { Triangular } \\
\text { C-DNM }\end{array}$ & $\begin{array}{c}\text { Rhombic } \\
\text { C-DNM }\end{array}$ \\
\hline Optimal frequency $(\mathrm{GHz})$ & 12.06 & 12.34 & 11.86 & 11.50 & 11.82 \\
\hline Reflection coefficient (dB) & -35.96 & -14.16 & -27.86 & -12.39 & -24.78 \\
\hline Maximal directivity (dB) & 7.78 & 7.79 & 8.02 & 8.01 & 7.82 \\
\hline Bandwidth (MHz) & 800 & 500 & 750 & 400 & 750 \\
\hline E-Plane HPBW (deg) & 73.90 & 74.70 & 73.80 & 73.70 & 75.60 \\
\hline H-Plane HPBW (deg) & 69.70 & 68.50 & 69.70 & 68.30 & 68.60 \\
\hline
\end{tabular}


Table 1 shows the comparison of the various performances between the normal patch antenna and the antenna incorporated with single C-DNM. From the graph, we notice a shift in the frequency resonant to a higher frequency for a C-DNM circular shape compared with the square patch antenna and a low frequency for other structure. For the reflection coefficient of C-DNM structure had decrease compared to the normal patch antenna, the same remark to the bandwidth. By incorporating the C-DNM structure, it improved the directivity gain to a value of $8.02 \mathrm{~dB}$ for square C-DNM structure compared to the normal patch antenna. Furthermore, the aperture at $-3 \mathrm{~dB}$ for the simulated square patch antenna in E-plane has reduced by $73.90 \mathrm{deg}$ to $73.80 \mathrm{deg}$ for square C-DNM and 73.70deg for triangular C-DNM. Unlike, there's a significant increase in other structure, such as circular and rhombic. The radiation pattern of the square patch antenna as a gain plot in H-plane is decreased from 69.70deg to the antenna alone to 68.30deg for triangular C-DNM, 68.50deg for circular C-DNM and 68.60deg with rhombic C-DNM. Hence, we conclude that the antenna is more directives.

\section{CONCLUSION}

A comparative study of a double negative metamaterial structure incorporated into the square patch antenna is demonstrated. To obtain a best directivity, C-DNM is used which is in the various shapes of metamaterial and is placed in the center of the square patch antenna. From the simulation work using Ansoft HFSS software, the directivity gain of the patch antenna shown an increased compared to the antenna incorporated with C-DNM. The different C-DNM shapes improved the radiation pattern. This paper studies at the complementary DNM structure which was incorporated with a square patch antenna with various shapes to observe the improvement on the properties of the antenna.

\section{REFERENCES}

[1] V.G Veselago, The electrodynamics of substances with simultaneously negative values of $\varepsilon$ and $\mu$, Soviet Physics Uspekh. vol. 10, no.4, pp. 509, Jan - Feb. 1968.

[2] J.B PENDRY, Negative Refraction Makes a Perfect Lens, In Phys. Rev. Lett,vol. 85, No. 18, pages 3966_3969, 2000.

[3] D Steyaert, Nouvelles structures à bande interdite photonique pour applications antennaires, Master work, University of Bordeaux1, 2006.

[4] S Yahya, H Khraisat1, Melad M Olaimat2 and Sharief N. Abdel-Razeq, Comparison between Rectangular and Triangular Patch Antennas Arrays, Applied Physics Research Vol. 4, No. 2; 2012. 